\title{
Una rassegna bibliografica sui traduttori romeni dell'Inferno (1883-2015): considerazioni di tipo metodologico e deontologico*
}

\section{Iulia COSMA}

Università dell'Ovest di Timisoara

Romania

Riassunto: il presente contributo si iscrive in una ricerca più ampia, finalizzata alla realizzazione di una monografia traduttiva delle versioni romene dell'Inferno dantesco, dall'Ottocento fino al Duemila. Si tratta di un'impresa di carattere storico e criticointerpretativo, collocabile all'incrocio di varie discipline: storia della traduzione, critica della traduzione, teoria della ricezione, storia della letteratura, storia della lingua letteraria, storia culturale. Per quanto riguarda la raccolta bibliografica, intendiamo fornire non soltanto i dati, ma anche alcune considerazioni di tipo metodologico e deontologico, di utilità nello studio della storia della traduzione.

Parole chiave: storia della traduzione, traduttori dell'Inferno in romeno, metodologia della storia della traduzione, deontologia della storia della traduzione, monografia traduttiva.

\begin{abstract}
This paper is intended as part of a larger research that aims to the realization of a monographic study dedicated to the Romanian translations of Dante's Inferno, from $19^{\text {th }}$ to 21 th century. It is a historical and critical approach, intended as an interdisciplinary study, to be placed at the crossing of disciplines like translation history, translation criticism, reception theory, history of literature, history of literary language, cultural history. The bibliographical selection we propose is complete with some methodological and deontological considerations of utility in the study of the history of translation.
\end{abstract}

Kywords: translation history, Romanian translators of Dante's Inferno, methodology in translation history, deontology in translation history, translational monography.

\section{Premessa}

Il presente contributo si iscrive in una ricerca più ampia finalizzata alla realizzazione di una monografia traduttiva delle versioni romene parziali e integrali dell'Inferno dantesco, dall'Ottocento fino al

\footnotetext{
* This work was cofinanced from the European Social Fund through Sectoral Operational Programme Human Resources Development 2007-2013, project number POSDRU/159/1.5/S/140863, Competitive Researchers in Europe in the Field of Humanities and Socio-Economic Sciences. A Multi-regional Research Network.
} 
Duemila. Si tratta di un'impresa di carattere storico e criticointerpretativo, collocabile all'incrocio di varie discipline: storia della traduzione, critica della traduzione, teoria della ricezione, storia della letteratura, storia della lingua letteraria, storia culturale. La finalità di questa indagine è quella di rilevare l'attività traduttiva in romeno, mediante lo studio delle varie ritraduzioni di un' unica opera, estese lungo un periodo segnato da ideologie letterarie contrastanti e, in alcuni momenti, dalla censura. I risultati ottenuti potranno servire allo studio della storia della traduzione in romeno e della ricezione di Dante in Romania, così come allo studio della storia della letteratura romena, visto che tra i numerosi traduttori di Dante si annoverano poeti e poetesse, critici e docenti universitari, o alla comparatistica, alla quale potrebbe fornire nuovi spunti di riflessione.

\section{Questioni metodologiche e deontologiche}

La letteratura scientifica sulla storiografia della traduzione concorda, in linea di massima, sull'utilità degi studi bibliografici (Delisle 1997-1998, Pym 1998, Sabio Pinilla 2005), manifestando tuttavia delle riserve. In questo senso, Delisle attira l'attenzione sul pericolo reale di arrivare a confondere la mera raccolta di traduzioni o di biografie di traduttori con la storia della traduzione (1997-1998, 2143), mentre Pym insiste sulla necessità di non lasciarsi prendere dall'impulso di raccogliere materiali solo in apparenza attinenti al campo di ricerca, ma di basarsi su uno studio metodico $(1998,11)$ e a lungo termine, per avere dei risultati pertinenti e duraturi (13). Per Sabino Pinilla invece, qualsiasi documento potrebbe rivelarsi utile alla ricerca $(2005,42)$, motivo per cui le rassegne bibliografiche svolgono un ruolo strumentale di estrema necessità $(2005,32)$, sempre e quando lo storico adoperi dei criteri di selezione secondo la rilevanza relativa al proprio processo investigativo, e non si limiti alla semplice compilazione (42).

Nel tentativo di fornire dei dati utili alla ricerca della storia della traduzione dall'italiano al romeno e tenendo presente sia l'arguta osservazione di Pym sulla neutralità soltanto apparente dei cataloghi (42) sia l'impossibilità dell'esaustività, abbiamo ideato la nostra bibliografia come un corpus. Dato che l'intento era quello di mettere a disposizione dei ricercatori il risultato di un lavoro basato, inevitabilmente, su criteri personali di selezione, abbiamo cercato di includere, per quanto possibile, un'ampia categoria di documenti, registrando tutti quelli in cui venivano fatti dei riferimenti ai traduttori dell'Inferno dantesco: cronache delle traduzioni, sia sulla stampa tradizionale sia on line, nell'eventualità in cui il titolare del blog fosse un italianista e avesse un minimo di visibilità ${ }^{1}$, interviste, contributi

${ }^{1}$ È il caso di Laszlo Alexandru, Răzvan Codrescu e George Popescu. 
sparsi in riviste sia su formato cartaceo che elettronico, libri, dizionari, storie della letteratura, introduzioni alle traduzioni. Una volta avviata la ricerca, abbiamo stabilito i seguenti elementi discriminatori: a) gli autori dovevano essere romeni, il loro contributo poteva essere in una lingua straniera, ma doveva risultare reperibile al pubblico romeno, visto che ci interessava la ricezione delle traduzioni da parte degli intellettuali romeni; b) nel contributo dovevano essere presenti considerazioni di tipo critico, descrittivo o prescrittivo sull'attività traduttiva, quindi abbiamo escluso le voci su Ion Heliade Rădulescu, Gheorghe Asachi, George Coşbuc, Nicolae Gane, Eta Boeriu o Marian Papahagi di alcuni dizionari e storie della letteratura romena, in cui veniva specificato soltanto il loro statuto di traduttori dell'Inferno o della Commedia, in quanto prive di valore conoscitivo; c) abbiamo escluso i documenti redatti in prima persona da alcuni traduttori contenenti motivazioni personali e spiegazioni delle proprie scelte traduttive. Abbiamo in fine optato per una disposizione di tipo cronologico, a differenza di Veronica Turcuş, nella cui rassegna bibliografica su Alexandru Marcu è stato adoperato il criterio alfabetico (2004, 267-316), del tutto privo di funzionalità. Per facilitarne la consultazione, abbiamo diviso la rassegna tenendo presente la data di stampa della prima traduzione integrale ${ }^{2}$ di Maria Chițiu 1883, anche se in prosa, e le seguenti ritraduzioni in versi che hanno suscitato maggiore interesse nell'ambiente culturale romeno al momento della pubblicazione ed in seguito: George Coșbuc 1925, edizione postuma, Alexandru Marcu 1932, Eta Boeriu 1965, Marian Papahagi 2012, edizione postuma. Abbiamo ritenuto utile menzionare tra parentesi quadre, laddove non fosse specificato o non risultasse dal titolo, il traduttore/i traduttori le cui versioni venivano prese in discussione.

L'inevitabile omissione dalla raccolta bibliografica di alcuni lavori non conformi ai criteri di selezione ci ha spinti a fare alcune riflessioni di tipo deontologico: avremmo dovuto specificare, almeno in nota, di averli consultati? E nel caso di una risposta positiva - in definitiva costituivano parte della ricerca-, tale menzione avrebbe servito o meno ai fini della nostra indagine? In riferimento a quanto riportato nelle considerazioni iniziali, la nostra rassegna intende superare lo statuto di mera compilazione, pur non screditando il proprio impegno conoscitivo e selettivo, e di fornire al lettore gli estremi per una chiave di lettura, da prendere in considerazione o meno, nello sviluppo di una futura ricerca. $\grave{E}$ in quest'ottica che abbiamo sentito la necessità di proporre alla fine anche una risposta al

2 Versioni parziali sono state realizzate da I.H. Rădulescu (1848), G. Asachi (1865), A. Densusianu (1865), G. H. Grandea (1870), I. C. Drăgescu (1877), Gr.Sc. Grădișteanu (1881), N. Gane (1882), M. P. Chițu (1882), I. H. Rădulescu (1893), G. Boteanu (1893-1894), G. Coșbuc (1900). 
quesito sull'(in)utilità di alcuni contributi, in relazione al corpus individuato.

\section{Rassegna bibliografica}

Gli studi da noi identificati sono, per quanto riguarda l'Ottocento e gli anni 30 del Novecento, soprattutto cronache di traduzioni. Per avere dei contributi di tipo storiografico bisogna aspettare gli anni 6o, più precisamente il 1965, quando, in vista della ricorrenza dei 700 anni dalla nascita di Dante, compaiono studi sparsi in riviste e volumi collettivi. La parte più corposa della nostra rassegna è costituita da dizionari e storie della letteratura, dato che la maggior parte dei traduttori di Dante sono stati poeti o narratori di fondamentale o di una qualche importanza nella storia della lingua e della letteratura romene. Tuttavia, come abbiamo precisato in precedenza, non tutti gli storici della letteratura mettono nel giusto risalto l'attività traduttiva degli scrittori coinvolti nella traduzione dell'Inferno. Riproponiamo, in seguito, il nostro corpus, disposto cronologicamente:

\section{3 $^{3-1923}$}

Dangely [Demetriescu, Anghel], «La Divina Commedia, Inferno, traducere de doamna Maria P. Chițu», in Voința Națională, a. I, no. 71, 5 ottobre, 1884: 1; no. 72, 6 ottobre, 1884: 1; no. 73, 7-8 ottobre 1884: 1 ; no. 74, 9 ottobre, 1884 : 1 .

Demetrescu, Traian, «Note bibliografice», in Revista Olteană, Craiova, 1888: 174-176. [M. Chițu]

Demetrescu, Traian, «Maria P. Chițu», in Profile literare, Craiova, Editura Tipografiei D. I. Benvenisti, 1891: 143-147.

Strajan, Nicolae, «Despre Dante», in Convorbiri literare, 1 luglio, no. XXIV, 1890: 290 [trad. di M. Chițu]

Caion [Constantin Al. Ionescu], «Monstruozități polygloto-coșbuciste în traducția „Infernului” lui Dante», in Forța morală, terza edizione, 17 febbraio, 1902: 3 .

Demetriescu, Anghel, "Opera lui Dante», in Voința Națională, seconda edizione, 31 luglio (13 agosto), 1903: 1. [trad. di M. Chițu]

Xenopol, A. D., «Dante Alighieri. Divina Comedie. Infernul. Traducere în versuri de N. Gane», in Arhiva, Iași, a. XVII (1906): 214-225.

Mavrocordato, Alexandru G., "Dante», in Arhiva, Iași, a. XVII (1906): 421-440, 486-497. [trad. di N. Gane]

B., P., «Recenzii. N. Gane: Dante Aligheri: Divina Comedie, Infernul», in Viața Românească, Iași, no 3, maggio, 1906: 511-512.

Iorga, Nicolae, «Dante în românește. Traducerea d-lui Gane», in Floarea Darurilor, București, no. 34, 18 novembre 1907: 529-530.

Anon., in Tribuna, Arad a. XII, no.7, 20 giugno 1908: 1-2. [cronaca della terza edizione della traduzione di Gane]

3 Per il periodo 1883-1931 ci siamo avvalsi dello studio di Pârvulescu e Panaitescu, Dante în România, pubblicato nel 1965. 
Ionescu, Constantin, «O carte genială în românește», in Datina, Turnu Severin, dicembre 1924: 232-238. [trad. di G. Coșbuc]

1925-1931

Bucuța, Emanoil, «Pe urme de terține dantești», in Mișcarea literară, 17 gennaio, 1925: 2. [trad. di G. Coșbuc]

Struțeanu, Scarlat, «Comentarii critice», in Mișcarea literară, 17 gennaio, 1925: 3 [trad. di G. Coșbuc]

Crețu, N. N., [recensione alla traduzione di Coșbuc], in Ritmul Vremii, marzo, 1925, pp. 40-42.

Iorga, Nicolae, “„Infernul”» lui Dante, in Neamul românesc literar, 1 gennaio, 1926: 2. [trad. di G. Coșbuc]

Buzdugan, C. Z., «O nouă traducere în românește a „Divinei Comedii” de Dante», in Adevărul literar și artistic, 24 febbraio, 1929: 5. [trad. di G. Coșbuc]

Anon., in Viața literară, 13 aprile, 1929: 4. [La redazione interviene in difesa di Coşbuc, criticato da Buzdugan.]

\section{4-1964}

Bucuța, Emanoil, «Săptămâna lui Coșbuc», in Vremea, 28 febbraio, 1932: 8. Anon., «O nouă ediție românească a lui Dante» in Secolul, București, a. I, no. 2, 21 febbraio, 1932: 7. [trad. di Al. Marcu]

Călinescu, George, [cronaca della traduzioni di M. Chițu, N. Gane], in Adevărul literar şi artistic, 6 marzo 1932.

Fortunescu, Constantin D., «Infernul lui Dante, în traducerea d-lui Alex. Marcu, cu il. de Mac Constantinescu, Edit. Scrisul Românesc, Craiova, 1932», in Arhivele Olteniei, Craiova, a. XI, no. 59-6o, gennaio-aprile 1932: 117.

Busuioceanu, Al., "O traducere nouă a lui Dante», in Secolul, București, a. I, no. 8, 3 aprile 1932: 4 [le traduzioni in romeno dell'Inferno di Al. Marcu e del Paradiso di Coșbuc]

Ionescu, Constantin D., «Despre a patra tălmăcire românească a Infernului: versiunea Alexandru Marcu», in Gândirea, Bucureşti, a. XII, no. 4, aprile 1932: 185-186.

Anon., "O carte de artă», in România Literară, București, a. I, no. 8, 9 aprile, 1932: IV. [segnalazione della trad. di Al. Marcu]

Anon., «Dante: Infernul», in Adevărul literar și artistic, București, serie II, a. X, no. 593, 17 aprile 1932: 5 [trad. di. Al. Marcu]

Gulian, Emil, «Dante - Infernul. Tradus de Alexandru Marcu, ilustrat de Mac Constantinescu. Editura "Scrisul Românesc"», in România Literară, București, a. I, no. 10, 23 aprile 1932: III.

Schintee -Seuca, I. N., "Dante: Infernul (tradus de Alex. Marcu, ilustrat de Mac Constantinescu), Scrisul Românesc, Craiova», in Ion Maiorescu. Revista elevilor Liceului «Carol I», Craiova, a. II, no. 3-4, marzo-aprile 1932: 94-95.

Struțeanu, Scarlat, «Coşbuc - „Divina Commedie” », in Convorbiri literare, marzo-aprile, 1932: 325-328.

4 I riferimenti bibliografici relativi all'attività di Alexandru Marcu pubblicati nel periodo 1932-1937 sono stati ripresi dal libro di Veronica Turcuș, Alexandru Marcu 1894-1955. Viața și opera, del 2004. 
Anon., «Dante - Infernul: Traducere de Al. Marcu - Scrisul Românesc, Craiova», in Cele trei Crișuri. Revistă de cultură, Oradea, a. XIII, no. 7-8, luglio-agosto 1932: 80.

Bucuța, Emanoil, «Un nou Dante românesc», in Boabe de Grâu, București, a. III, no. 3-4, marzo-aprile 1932: 110 [trad. di Al. Marcu] (Struțeanu, Scarlat, «Dante: Infernul, traducere de Alexandru Marcu, Scrisul Românesc, Craiova», in Convorbiri literare, București, a. LXV, maggio-luglio, 1932: 449451.

Şerban Cioculescu, "Anul literar. Traducerile», in Adevărul, București, a. XLVII, no. 15031, 1 gennaio 1933: 7 [trad. di Al. Marcu].

A.B., "Traducerile», in Facla, București, a. XII, no. 665, 15 aprile 1933: 2 [trad. di Al. Marcu].

Anon., «Traduceri» in Glasul Bucovinei, Cernăuți, a. XVI, no. 4050, 30 aprile 1933: 3. [trad. di Al. Marcu]

Herescu, N. I., "Dante în românește», in Pleiada. Revistă trimestrială pentru poezie și artă, București, a. I, gennaio-marzo 1934: 64-72. [trad. di Al. Marcu]

Robot, Al., "Traduceri», in Rampa nouă ilustrată, București, a. XVII, no, 4800, 13 gennaio 1934: 1 [trad. di Al. Marcu]

Anon., «Cronica măruntă» in Azi, București, a. IV, no. 1, gennaio-aprile 1935: 1602 [trad. Al Marcu].

Georgescu, Horia, «Dante în românește. Pe marginea traducerii D-lui Alexandru Marcu», in Gând Românesc, Cluj, a. III, no. 4, aprile 1935: 243245 .

Schintee-Seuca, I., "Alexandru Marcu, traducător al lui Dante», in Ramuri, Craiova, a. XXVII, no. 7-9, luglio-dicembre 1935: 491-493.

Damaschin, Vasile, "Ceva despre tălmăcirile d-lui Al. Marcu», in Azi, București, a. IV, no. 2, maggio 1935: 1657-1662.

Şuluțiu, Octav, «Traducerile și cultura română», in Da și nu, București, a. I, no. 1, 5 marzo 1936: 4. [trad. di Al. Marcu]

Roșu, Nicolae, "Glosse actuale», in Pagini Literare, Turda, a. IV, no. 6-8, 1937: 313-317. [trad. di Al. Marcu]

Iorga, Nicolae, «V. Alecsandri ca traducător al lui Dante», in Cuget Clar, București, 31 marzo 1938: 593-594.

Iorga, Nicolae, "Prefață», in Dante Alighieri Divina Comedie traducere de Ioan A. Țundrea, București, Editura Casa Școalelor, 1945: 5.

Balaci, Alexandru, Studii italiene, București, Editura de Stat pentru Literatură și Artă, 1958: 47. [trad. di Coșbuc].

Duțu, Alexandru, "Alegorie și realism în „Divina Comedie” - însemnări inedite ale lui Coşbuc», in Studii de literatură universală, București, vol. II, Editura științifică, 1960: 141-149.

Vianu, Tudor, "Coșbuc, traducător al lui Dante», in Studii de literatură universală şi comparată, seconda edizione, București, Editura Academiei Republicii Populare Romîne, 1963: 597-600.

1965-2011

Pârvulescu, Titus, Panaitescu, Dumitru D., "Dante în România», in Studii despre Dante, București, Editura pentru Literatură Universală, 1965: 345-422. [I. H. Rădulescu, M. Chițu, N. Gane, G. Coșnuc, Al. Marcu, I. Țundrea, menzione del fatto che Eta Boeriu stava lavorando ad una nuova versione]. 
Pătrașcu, Ion, «Divina Comedia în românește», in Ramuri, a. II, no. 5(10), 15 maggio 1965: 13. [I. H. Rădulescu, M. Chițiu, N. Gane, G. Coșbuc]

Şchiopu, Mihaela, "Aspecte ale recepției operei lui Dante în România», in Revista de istorie și teorie literară, București, no. 1, 1965: 133-148. [I. H. Rădulescu, G. Asaky, Aron Densusianu, M. Chițiu, N. Gane, G. Coșbuc]

Constantinescu, Pimen, «Atitudinea lui Coşbuc faţă de traducerea Divinei Comedii» in Limbă şi literatură, Bucureşti, vol. XI, 1966: 17.

Balaci, Alexandru, "Opera de traducător a lui George Coșbuc», in Extras din Analele Academiei Rpublicii Socialiste România, București, a. C, serie IV, volume XVI, 1966: 649-654.

Călinescu, George, "Ion Heliade-Rădulescu», in Istoria literaturii române II De la Școala Ardeleană la Junimea București, Editura Academiei Republicii Socialiste România, 1968: 261-294.

Doinaş, Ştefan Augustin, Poezie și modă poetică, București, Editura Eminescu, 1972: 299 [trad. di Eta Boeriu].

Scridon, Gavril, "George Coșbuc», in Istoria literaturii române III Epoca marilor clasici, București, Editura Academiei Republicii Socialiste România, 1973: 759-814.

Piru, Alexandru, «Coşbuc traducător și tradus», in Reflexe şi interferențe, Craiova, Editura Scrisul Românesc, 1974: 187-193.

Firan Florea, «Maria P. Chițu», in De la Macedonski la Arghezi, Craiova, Editura Scrisul Românesc, 1975: 114-115.

Crețu, Stănuța, "Maria Chițu», in Dicționarul literaturii române de la origini până la 1900, București, Editura Academiei Republicii Socialiste România, 1979: 180-181.

Crețu, Stănuța, "George Coșbuc», in Dicționarul literaturii române de la origini până la 1900, București, Editura Academiei Republicii Socialiste România, 1979: 228-233.

Zăstroiu, Remus, «Ion Heliade-Rădulescu», in Dicționarul literaturï române de la origini până la 1900, București, Editura Academiei Republicii Socialiste România, 1979: 432-437.

Niculescu, Alexandru, "Arta traducerii poetice în poezia românească de astăzi», in Între filologie și poetică, București, Editura Eminescu. 1980: 78-82 [Eta Boeriu, George Coșbuc, Alexandru Marcu, George Buznea].

Piru, Alexandru, "George Coșbuc», in Istoria literaturii române de la început până astăzi, București, Editura Univers, 1981: 179-182.

Ionescu, Gelu, Orizontul traducerii, București, Editura Univers, 1981: 41. [G. Coșbuc]

Călinescu, George, «I. Eliade Rădulescu », in Istoria literaturii române de la origini până în prezent, Ediție și prefață de Al. Piru, seconda edizione rivista ed ampliata, București, Editura Minerva, 1982: 131-149.

Călinescu, George, "George Coșbuc», in Istoria literaturii române de la origini până în prezent, Ediție și prefață de Al. Piru, seconda edizione rivista ed ampliata, București, Editura Minerva, 1982: 585-590.

Călinescu, George, "Nicolae Gane», in Istoria literaturii române de la origini până în prezent, Ediție și prefață de Al. Piru, seconda edizione rivista ed ampliata, București, Editura Minerva, 1982: 431-432.

Cordoneanu, Maria, George Coşbuc interpretat de Maria Cordoneanu; Prefaţă, antologie, note, tabel cronologic şi bibliografie selectivă de Maria Cordoneanu, București, Editura Eminescu, 1982: 447-460. 
Duțu, Alexandru, «Prefață», in George Coşbuc Opere alese, vol. VII Traduceri, București, Editura Minerva, 1985: pp. vii-xx.

Chivu, Marius, "Manuscrisele Divinei Comedii. Elemente pentru o cronologie a traducerii datorate lui George Coșbuc», », in George Coşbuc Opere alese, vol. VII Traduceri, București, Editura Minerva, 1985: xxi-xliii.

Iorga, Nicolae, "George Coșbuc», in Istoria literaturii românești contemporane I, București, Editura Minerva, 1986: 384-389.

Negoițescu, Ion, "George Coșbuc», in Istoria literaturii române vol. I (180o-1945). București: Editura Minerva, 1991: 135-138.

Ciorănescu, Alexandru, "Cuvânt înainte», in Dante Alighieri Divina Comedie în românește de Giuseppe Cifarelli, îngrijire de ediție, note și comentarii de Titus Pârvulescu, Craiova, Editura Europa, 1993: 5-6.

Popescu, Adrian, «Lectura Dantis», in Steaua, a. XLIV, no. 6, iunie 1993: 31-32. [G. Cifarelli, E. Boeriu]

Duțu, Alexandru, «În dialog cu literaturile europene», in Jurnalul literar, a. V, no. 9-12, aprilie 1994: 3. [cronaca della traduzione di G. Cifarelli]

Rotaru, Ion, «George Coșbuc», in O istorie a literaturii române vol III De la Junimea până după primul război mondial, Galați, Editura Porto-Franco, 1996: 266-278.

Bantaș, Andrei, Croitoru, Elena, «Istoricul traducerilor în România», in Didactica traducerii, București, Editura Teora, 1998: 107-108. [G. Coșbuc]

Micu, Dumitru, «George Coşbuc», in Istoria literaturii române, București, Editura Saeculum I.O., 2000: 163-165.

Papahagi Marian, «Eta Boeriu», in Dicționarul scriitorilor români vol. 1 AC, București, Editura Fundației Culturale Române, 1995: 303-305.

Mihăilescu, Călin A., "Alexandru Marcu», in Dicționarul scriitorilor români, vol. 2 M-Q, București, Editura Albatros, 2001: 72-75.

Crețu, Stănuța, "George Coşbuc», in Dicționarul general al literaturii române Vol. C/D, București, Editura Univers Enciclopedic, 2004: 407-415.

Grasso, Dana, Traduttologia e traduzione. Nozioni teoriche e applicazioni pratiche/Noțiuni teoretice și aplicații practice. Traductologie și traducere, București, Editura Meteor Press, 2003: 243-248. [G. Cossbuc, E. Boeriu]

Ionescu, Gelu, Orizontul traducerii, seconda edizione rivista, București, Editura Institutului Cultural Român, 2004: 38-39. [G. Coșbuc, E. Boeriu]

Popescu, Corina, «Eta Boeriu», in Dicționarul general al literaturii române Vol. A/B, București, Editura Univers Enciclopedic, 2004: 571-573.

Turcuș, Veronica, Alexandru Marcu (1894-1955). Viața și opera, București, Editura România Press, 2004: 83-89.

Manu, Emil, «Prima traducere din Dante în românește», in Luceafărul, no. 27 (704), 13 luglio, 2005: 23. [Maria P. Chițu]

Popa, Ciprian, "Culpabilizarea traducerii în alterarea mesajului poetic românesc", in Analele Universității "Ștefan cel Mare din Suceava, Seria Filologie. A Lingvistică, a. 11, no. 1, 2005: 191-194.[G. Coșbuc, G. Buznea, E. Boeriu]

Volovici, Leon, «Ion Heliade-Rădulescu», in Dicţionarul general al literaturii române E/K, București, Editura Univers Enciclopedic, 2005: 486496. 
Alexandru, Laszlo, «Marian Papahagi: portret neretuşat»5, in Tribuna, Cluj, no. 75/16-31 ottobre 2005: 10-11.

Alexandru, Laszlo, «Italienistul Alexandru Marcu» 6 , in Tribuna, Cluj, no. 77/16-30 novembre 2005: 9-10.

Alexandru, Laszlo, «Dante tradus de Eta Boeriu»7, in Tribuna, Cluj, no. 88/1-15 maggio 2006, p. 18.

Alexandru, Laszlo, Pecican, Ovidiu, «Dialoguri despre Dante (I) ${ }^{8}$, in Vatra, Tîrgu Mureş, no. 8 (425)/agosto 2006: 57-67. [G. Coşbuc, Al. Marcu, I. Țundrea, E. Boeriu, G. Cifarelli]

Jeanrenaud, Magda, Universaliile traducerii. Studii de traductologie, Iași, Editura Polirom, 2006: 191-192. [G. Coșbuc]

Sasu, Aurel, «Eta Boeriu», in Dicționarul biografic al literaturii române, Vol. I, Pitești, Paralela 45, 2006: 178-179.

Spiridon, Monica, «Marian Papahagi», in Dicționarul general al literaturii române, Vol. P/R, București, Editura Univers Enciclopedic, 2006: 69-71.

Stanca, Dan, «Dante, din nou în românește»9, in Aldine, 9 dicembre 2006: 1 [trad. di R. Codrescu].

Vasileanu, Marius, «Divina Comedie, o nouă versiune», in Adevărul, 16 gennaio 2007: 12 [trad. di R. Codrescu].

Baconsky, Theoror, «Infernul regăsit», in Dilema Veche, a. IV, no. 154, 1925 gennaio 2007, p. 18 [trad. di R. Codrescu].

Alexandru, Laszlo, "Coşbuc şi Dante» ${ }^{10}$, in Tribuna, Cluj, no. 105/16-31 gennaio 2007, p. 9-10; no. 106/1-15 febbraio 2007: 8.

Platon, Mircea, "Nostalgii înviorătoare», in Convorbiri literare, febbraio 2007 e in Rost, a. V, no. 49, marzo 2007: 51-52 [trad. di R. Codrescu].

Anon., «Eveniment editorial», in Credinţa ortodoxă, febbraio 2007: 3 [trad. di R. Codrescu].

Pop, Ioan Es., «Ultima carte pe care am citit-o: Infernu»l, in Descoperă, a. V, no. 8(48), settembre 2007: 108 [trad. di R. Codrescu].

Purcaru, Alina, "Cărţile ignorate ale verii», in Cotidianul, 15 settembre 2007, rubrica Arte [trad. di R. Codrescu].

Alexandru, Laszlo, Pecican, Ovidiu, «Dialoguri despre Dante (II)» ${ }^{11}$, in Acolada, Satu Mare, no. 1/ottobre 2007: 13; no. 2/novembre 2007: 8-9; no.

5 Disponibile on line: http://193.226.7.140/ laszlo/eleonardo/no8/ Laszlo7.htm.

6Disponibile on line: https://laszloal.wordpress.com/2012/02/12/italienistul alexandru-marcu-1/;https://laszloal.wordpress.com/2012/02/13/italienistul alexandru-marcu-2/;https://laszloal.wordpress.com/2012/02/14/italienistulalexandru-marcu-3/;https://laszloal.wordpress.com/2012/02/15/italienistul alexandru-marcu-4/.

7 Disponibile on line: http://193.226.7.140/ laszlo/eleonardo/n1o/ Laszlog.htm.

8 Disponibile on line: http://193.226.7.140/ laszlo/eleonardo/ no9/Dante1.htm.

9 Disponibile on line: http://www.romanialibera.ro/aldine/history/dante-din-nou-in-romaneste--24219.

10 Disponibile on line: http://193.226.7.140/ laszlo/eleonardo/ n11/Laszlo6.htm.

11 Disponibile on line: http://193.226.7.140/ laszlo/eleonardo/n1o/

Dante2.htm. 
3/dicembre 2007: 8. [I. H. Rădulescu, A. Densusianu, M. Chițu, N. Gane, G. Buznea, G. Coșbuc]

Mihăilescu, Dan C., «Pe ce filme trăim. Cărţile lui 2007», in Idei în Dialog, a. IV, no. 1/40, gennaio 2008: 7 [trad. di R. Codrescu].

Cărcăleanu, Eleonora, «Divina Comedie în echivalări românești», in Convorbiri literare, a. CXLI, no.1, gennaio 2008: 92-94. [G. Cifarelli, G. Coşbuc, Al. Marcu, E. Boeriu, M. Papahagi, R. Codrescu].

Manolescu, Nicolae, «Ion Heliade-Rădulescu», in Istoria critică $a$ literaturii române. 5 secole de literatură, Pitești, Editura Paralela 45, 2008: 169-192.

Manolescu, Nicolae, "George Coșbuc», in Istoria critică a literaturii române. 5 secole de literatură, Pitești, Editura Paralela 45, 2008: 503-508.

Virgil, Vintilescu, «George Coșbuc. Traducerile», in Istoria literaturii române „Eроса Junimii”, Timișoara, Editura Excelsior Art, 2008: 1022-1029.

Tomi, Raluca, «Una traduttrice meno conosciuta di Dante: Maria Chițu e gli echi europei delle sue traduione della Divina Commedia» ${ }^{12}$, in Historical Yearbook, București, vol. VII, 2010: 77-92.

Zamfir, Mihai, "George Coșbuc. Nostalgia Clasicismului», in Scurtă istorie: panorama alternativă a literaturii române, vol. 1, Editura Cartea Românească, 2011: 450-464.

\section{2-2015}

Mocan, Mira, «Introducere», in Dante Alighieri Infernul, traducere din italiană şi comentarï Marian Papahagi, Ediție îngrijită, introducere și completarea comentariilor de Mira Mocan, București, Editura Humanitas, 2012: 19-40.

Papahagi, Irina, «Prefață», in Dante Alighieri Infernul, traducere din italiană şi comentarii Marian Papahagi, Ediție îngrijită, introducere și completarea comentariilor de Mira Mocan, București, Editura Humanitas, 2012: 7-17.

Alexandru, Laszlo, «Şantier dantesc»13, in Bucovina literară, Suceava, no. 1-2/gennaio-febbraio 2012: 74-75; no. 3-4/marzo-aprile 2012: 62-63.

Codrescu, Răzvan, «Dantele lui Marian Papahagii», 28 marzo 2012, pubblicato sul blog personale: http://razvancodrescu.blogspot.ro/2012/o3/dantele-lui-marian-papahagi.html

Tartler, Grete, «Transparentă, aspră, vie: traducerea Infernului în hermeneutica lui Marian Papahagi», in România literară, no. 17, 27 aprile- 3 maggio $2012^{14}$.

Dumbravă, Daniela, «„Infernul”, nouă ediţie critică. Dialog cu Mira Mocan şi Corrado Bologna»15, in Orizonturi culturale italo-române/Orizzonti culturali italo-romeni, a. II, no. 7, luglio 2012.

12 Disponibile on line: http://www.iini-minorities.ro/resurse/TomiRaluca_Una-traduttrice_2010.pdf.

13 Disponibile on line: http://193.226.7.140/ laszlo/eleonardo/n18/ Laszlo12.htm.

14 Disponibile on line: http://www.romlit.ro/transparent_aspr_vie_ traducerea_infernului_n_hermeneutica_lui_marian_papahagi.

15 Disponibile on line anche in versione italiana: http://www.orizonturiculturale.ro/ro_intalniri_Corrado-Bologna-si-MiraMocan-interviu.html. 
Crețu, Stănuța, «George Coșbuc», in Dicționarul literaturii române, București, Univers Enciclopedic Gold, 2012: 395-400.

Călina, Nicoleta, «On the first woman translator of Dante's 'The Divine Comedy' into Romanian» ${ }^{16}$, in Iulian Boldea (coord.), Studies on Literature, Discourse and Multicutural Dialogue. Section: Language and Discourse, Târgu Mureş, Editura Arhipelag XXI, 2013: 818-826.

Braniște, Ludmila, Petrovici Oana Maria, «Lyrical Expressivity in Romanian and the Art of Translation»17, in European Integration-between tradition and modernity, no. 5, 2013: 588-595. [trad. di G. Coșbuc]

Voncu Răzvan, "Coşbuc pe şantierul Divinei Comedii»18, in România literară, no. 9, 28 febbraio-6 marzo 2014.

Anon., «RAO clasic/ de Ziarul de duminică»19, in Ziarul Financiar, 17 gennaio, 2014. [menzione di varie traduzioni]

Cosma, Iulia, "L'influenza di Dante sull'enciclopedista romeno Ion Heliade Rădulescu e sull'italianismo in Romania» ${ }^{20}$, in Nasledje, XI, no. 29/2014, Casa Editrice della Facoltà di Filologia e Arti dell'Università di Kragujevac: 191-202.

Popescu, George, «Giuseppe Cifarelli - o „pagină” de re-cucerit a culturii române», 4 luglio 2015, pubblicazione on line sul blog personale: http://www.mondotexte.on.ro/o4.07.2015/giuseppe-cifarelli-\%E2\%80\%93\%E2\%80\%9Epagina\%E2\%80\%9D-de-re-cucerit-culturii-romane-2.

Chivu, Gheorghe, «Numele dracilor în traducerea românească a Divinei Comedii» 21, in Limba Română, Chișinău, a. XXV, no. 5-6, 2015: 249-256. [G. Coșbuc]

Cosma, Iulia, «Le prime traduzioni in romeno dell'Inferno dantesco: rigerimenti ulturali e traduttivi». In: Romània între interculturalitate şi identitate: Spaţii romanice europene şi extraeuropene. [coord.] C. Timoc. Szeged: University Press of Szeged, 2015. Gli Atti della terza edizione di CICCRE, 3 e 4 ottobre 2014: 413-421.

Cosma Iulia, «La prima traduttrice dell'Inferno dantesco in romeno: Maria Chitu», in via di pubblicazione negli Atti del Convegno internazionale "Traduzione e plurilinguismo", oraganizzato dal Dipartimento di Studi in lingua italiana dell'Università “Juraj Dobrila” di Pola, 3-4 luglio, 2015.

Una lettura attenta della rassegna bibliografica potrebbe fornire delle indicazioni preziose per quanto riguarda la storia della traduzione e la storia dell'italianistica in Romania. Come notavamo nella parte

16 Disponibile on line: http://www.upm.ro/ldmd/LDMD-01/Lds/ Lds\%2001\% 2092.pdf.

17 Disponibile on line: http://www.upm.ro/facultati_departamente/ stiinte_litere/conferinte/situl_integrare_europeana/Lucrari5/IETM5_Part7o. pdf.

18 Disponibile on line: http://www.romlit.ro/cobuc_pe_şantierul_divinei _comedii.

$\overline{19}^{-}$Disponibile on line: http://www.zf.ro/ziarul-de-duminica/rao-clasic-deziarul-de-duminica-11907761.

20 Disponibile on line: https://www.academia.edu/10129706/Linfluenza_di_ Dante_sullenciclopedista_romeno_Ion_Heliade_R\%C4\%83dulescu_e_sullita lianismo_in_Romania.

21 Disponibile on line: http://limbaromana.md/ index.php?go= articole\&n=3234. 
iniziale del nostro contributo, abbiamo riscontrato in alcune storie della letteratura e nella maggior parte dei dizionari letterari la mancanza di informazioni sull'attività di traduzione di Ion Heiade Rădulescu, Gheorghe Asachi, Nicolae Gane, George Coșbuc, Eta Boeriu o Marian Papahagi. Il caso di Alexandru Marcu è particolarmente interessante in quanto ostracizzato dal regime comunista per la sua attività culturale $\mathrm{e}$ politica filofascista. Tuttavia, l'assenza non è meno loquace della presenza, poiché potrebbe portare all'individuazione del valore attribuito all'attività traduttiva dagli autori di storie e dizionari della letteratura. Quindi il riferimento in nota a queste fonti diventa necessario ${ }^{22}$, nonostante l'esclusione dalla rassegna. La mancanza di

${ }^{22}$ In Șerban Cioculescu, Vladimir Streinu, Tudor Vianu (coord.), Istoria literaturii române moderne (1944) si registra l'attività di traduzione di Asachi, ma non ci sono delle considerazioni sulla traduzione frammentaria dall'Inferno, mancano accenni alle traduzioni di Heliade e per quanto riguarda Gane, si specifica soltanto che sia stato traduttore della Commedia; Valeriu Ciobanu, in Istoria literaturii române II De la Școala Ardeleană la Junimea (1968) menziona brevemente l'attività di traduzione di Asachi, senza rilevarne l'importanza; George Ivaşcu, in Istoria literaturii române vol. I (1969) registra il programma di traduzioni a cui intendeva dedicarsi Heliade, ma non si sofferma sulle sue traduzioni; Algeria Simota, nella voce dedicata ad Asachi in Dicționarul literaturii române de la origini până la 1900 (1979) sottolinea l'importanza delle sue traduzioni teatrali, ma non menziona la traduzione frammentaria dall'Inferno, così come in Dicționarul general al literaturii române A/B (2004); in Ion Negoițescu, Istoria literaturii române Vol. I 18001945 (1991) mancano i riferimenti a Nicolae Gane; in Mircea Zaciu, Marian Papahagi, Aurel Sasu (coord.), Dicționarul scriitorilor români A-C (1995) non viene dato il giusto risalto all'attività traduttiva di George Coșbuc, mentre nel Dicționarul scriitorilor români D-L (1998) e nel Dicționarul scrïtorilor români $M-Q$ (2001) a quella di Nicolae Gane e Alexandru Marcu; la versione ridotta, Dicționarul esențial al scriitorilor români (2002) presenta la stessa situazione; in Dicționarul general al literaturii române vol. L/O (2005), compare solo una breve menzione ad Alexandru Marcu traduttore, mentre dal volume $\mathrm{E} / \mathrm{K}$, mancano riferimenti all'attività di traduzione di HeliadeRădulescu o Gane; in Eugen Simion (coord.) Dicționarul literaturii române A$L$ e Dicționarul literaturii române $M-Z$ (2012) non si evidenzia l'importanza dell'attività di traduzine di Nicolae Gane, Eta Boeriu, Alexandru Marcu o Marian Papahagi. Per non parlare poi di Maria Chițu, la grande assente, menzionata soltanto da Dicționarul literaturii române de la origini până la 1900 (1979); Ion Rotaru, nella sua O istorie a literaturii române vol. II (1994), fa un accenno all'attività di traduzione di Heliade, mentre per quanto riguarda Asachi, ci sono dei riferimenti alle sue traduzioni dal russo; George Călinescu in Istoria literaturii române de la origini până în prezent (1982), seconda edizione rivista ed ampliata, non si sofferma più di tanto sull'attività traduttiva di Asachi; Virgil Vintilescu, nella sua Istoria literaturii române Epoca Junimii (2008) menziona la traduzione dell'Inferno da parte di Gane, mentre nell'altro volume, Istoria literaturii române Epoca modernă (2011), accenna brevemente all'attività di traduzione di Heliade, ma non menziona la traduzione frammentaria della prima cantica dantesca; Nicolae Manolescu, 
attenzione verso l'attività traduttiva degli scrittori in questione, potrebbe essere letta come sintomo di quella «contraddizione palese» 23 denunciata da Georgiana Lungu-Badea tra il riconoscimento, da parte degli studiosi romeni dell'importanza assunta dalla traduzione nella formazione di una letteratura nazionale e di un publico elevato culturalmente, e il loro disinteresse per la ricerca di tipo traduttivo e traduttologico $(2013,8)$. Al contrario, la presenza non indifferente di considerazioni relative alla traduzione nel caso di alcuni poeti, a nostro avviso non sarebbe indice di una valorizzazione in chiave positiva della traduzione letteraria, ma riguarda l'interesse dello storico ${ }^{24}$ della letteratura per l'attività traduttiva dell'autore studiato, testimoniata, nel caso di Al. Piru e Al. Duțu, da altri studi dedicati a Coşbuc traduttore. Grazie a questo, il poeta del mondo contadino - che non arriverà a vedere pubblicata la sua versione dell'Inferno -, si conquista il primato a livello di riferimenti bibliografici, di pari passo apprezzato e discusso.

La prima traduttrice di Dante in romeno, Maria Chițu, che firma le sue traduzioni con la variante latineggiante del cognome, Chițiu, dopo gli entusiasmi iniziali e le critiche severe degli anni sessanta (Șchiopu 1965; Pătrașcu 1965) è stata riscoperta da due studiose, Raluca Tomi (2010) e Nicoleta Călina (2013), forse sulla scia degli studi di genere. Per quanto ci riguarda, la sua svalutazione è conseguenza di approcci metodologici sbagliati, dell'applicazione di criteri di valutazione anacronistici ed estranei alla storiografia della traduzione.

La versione di Alexandru Marcu (1932) ha destato al momento della pubblicazione pareri contrastanti. Non possiamo non segnalare l'ipotesi che le opinioni negative siano attribuibili anche, se non soprattutto, a motivazioni di tipo politico, più esattamente ad animosità da parte di alcuni intellettuali vicini a Ramiro Ortiz, visto che sia la Turcuş (2004, 102-103), che la Derer (2007, 91, 93) accennano, da posizioni diverse e con atteggiamenti contrastanti, ad un conflitto tra i due, in seguito al quale il romenista sarebbe stato richiamato in patria.

\section{Conclusioni}

Il numero estremamente ridotto (circa l'1\% della nostra rassegna bibliografica) di studi dedicati all'analisi comparativa delle varie versioni della prima cantica dantesca in romeno conferma la

nella sua Istoria critică a literaturii române. 5 secole de literatură (2008), non registra la traduzione frammentaria dell'Inferno realizzata da Asachi; Mihai Zamfir si sofferma soltanto sull'attività di traduzione dal francese di Heliade Radulescu, nel suo Scurtă istorie: panorama alternativă a literaturii române (2011), mentre, nonostante egli rilevi l'interesse di Asachi per l'Italia e l'influnza della lirica petrarchesca sulla proprio modo di poetare, non fa alcun riferimento alle sue traduzioni dallitaliano.

23 Traduzione nostra.

${ }^{24}$ Al. Piru, G. Scridon, S. Crețu, Al. Duțu. 
mancanza d'interesse da parte degli italianisti per l'indagine di tipo critico e storico sulle traduzioni in romeno di autori italiani e rendono, a nostro avviso, necessaria la continuazione della ricerca in vista della realizzazione della nostra monografia traduttiva dell'Inferno in romeno. I risultati della ricerca monografica potranno servire sia a rilevare l'importanza dell'attività traduttiva di poeti ed intellettuali del secondo Ottocento e del primo Novecento romeno, sia a delineare i rapporti esistenti tra la prassi traduttiva e le ideologie linguistiche e letterarie, così come le politiche culturali vigenti nello spazio romeno tra la fine del XIX secolo e l'inizio del XXI.

\section{Riferimenti bibliografici}

Călinescu, George. «Gheorghe Asachi». In: Istoria literaturii române de la origini până în prezent, seconda edizione rivista ed ampliata. Bucarest: Editura Minerva, 1982: 94-110.

Ciobanu, Valeriu. «Gheorghe Asachi». In: Istoria literaturii române II De la Şcoala Ardeleană la Junimea. București: Editura Academiei Republicii Socialiste România, 1968: 354-372.

Delisle, Jean. «Réflexions sur l'historiographie de la traduction et ses exigences scientifiques». In: Équivalences (ISTI, Bruxelles), vol 26, no. 2 e vol. 27, no. 1, 1997-1998: 21-43.

Dicționarul scriitorilor români A-C. Zaciu, Mircea, Papahagi, Marian, Sasu, Aurel (coord). București: Editura Fundației Culturale Române, 1995.

Dicționarul scriitorilor români D-L. Zaciu, Mircea, Papahagi, Marian, Sasu, Aurel (coord). București: Editura Fundației Culturale Române, 1998.

Dicționarul scriitorilor români $M-Q$. Zaciu, Mircea, Papahagi, Marian, Sasu, Aurel (coord). București: Editura Albatros, 2001.

Dicționarul esențial al scrïtorilor români. Zaciu, Mircea, Papahagi, Marian, Sasu, Aurel (coord). București: Editura Albatros, 2000.

Dicționarul general al literaturii române $E / K$. București: Editura Univers Enciclopedic, 2005.

Derer Condrea, Doina. «Cuvânt înainte». In Doina Condrea Derer. Ramiro Ortiz- Nina Façon Carteggio - Corespondență. Ediție îngrijită, cuvânt înainte, traducere, tabel cronologic şi note de Doina Condrea Derer. București: Editura Jurnalul literar, 2007: 89-98.

Grigorescu, Dan. «Alexandru Marcu». In: Dicționarul general al literaturii române, Vol L/O. București: Editura Univers Enciclopedic, 2005: 221-223.

Istoria literaturii române moderne. Cioculescu, Serban, Streinu Vladimir, Vianu, Tudor (coord.). București: Editura Casei Școalelor, 1944.

Ivaşcu, George. «Părintele literaturii române: Ion Heliade-Rădulescu». In: Istoria litearturii române vol. I. București: Editura Științifică, 1969: 397-406. Lungu-Badea. Georgiana. Idei şi metaidei traductive românești (secolele XVIXXI). Timișoara: Editura Eurostampa, 2013.

Manolescu, Nicolae. "Gheorghe Asachi». In: Istoria critică a literaturii române. 5 secole de literatură. Pitești: , Editura Paralela 45, 2008: 158-166.

Pătrașcu, Ion. «Divina Comedie în românește». In: Ramuri, 5(10), 1965: 13.

Pârvulescu, Titus, Panaitescu, Dumitru. «Dante în România». In: ***Studii despre Dante. București: Editura pentru Literatură Universală, 1965: 345-422. Pym, Anthony. Method in Translation History. New York: Routledge, 1998. 
Repertoriul traducătorilor români de limbă franceză, italiană, spaniolă (secolele al XVIII-lea şi al XIX-lea). Studii de istorie a traducerii (II) LunguBadea, G. (coord.). Timişoara : Editura Universităţii de Vest, 2006.

Rotaru, Ion. «Ion Heliade-Rădulescu». In: $O$ istorie a literaturii române vol. II De la Epoca luminilor până la Junimea, seconda edizione rivista. Galați: Editura Porto-Franco, 1994: 145-169.

Rotaru, Ion. "Gheorghe Asachi». In: O istorie a literaturii române vol. II De la Epoca luminilor până la Junimea, seconda edizione rivista. Galați: Editura Porto-Franco, 1994: 159-164.

Sabio Pinilla, José Antonio. «La metodología en historia de la traduction: estado de la question». In: Sendebar, 17, 2006: 21-47.

Simota, Algeria. «Gheorghe Asachi». In: Dicționarul literaturii române de la origini până la 190o. București: Editura Academiei Republicii Socialiste România, 1979: 55-60.

Simota, Algeria. "Gheorghe Asachi». In: Dicționarul general al literaturii române. București: Editura Univers Enciclopedic, 2004: 252-257.

Șchiopu, Mihaela. «Aspecte ale receptiei operei lui Dante in Romania». In: Revista de istorie si teorie literară 14 (1), 1965: 133-149.

Turcuş, Veronica. Alexandru Marcu (1894-1955) Viața şi opera. București: Editura România Press, 2004.

Vintilescu Virgil. «Gerge Gane». In: Istoria literaturii române Epoca junimii.Timişoara: Editura Excelsior Art, 2008: 74-78.

Vintilescu Virgil. «Ion Heliade-Rădulescu». In: Istoria literaturii române Epoca modernă. Timișoara: Editura Excelsior Art, 2011: 108-162.

Zamfir, Mihai. «Ion Heliade Rădulescu». In: Scurtă istorie: panorama alternativă a literaturii române, vol. 1. București: Editura Cartea Românească, 2011: 58-66.

Zamfir, Mihai. «Gheorghe Asachi». In: Scurtă istorie: panorama alternativă a literaturii române, vol. 1. București: Editura Cartea Românească, 2011: 6775 . 